\title{
APPLICATION OF MATHEMATICAL METHODS FOR CURRENT DIAGNOSTICS OF CYLINDER-PISTON GROUP ELEMENTS OF DIESEL ENGINES
}

\author{
Aleksandrs Gasparjans ${ }^{1}$, Aleksandrs Terebkovs ${ }^{1}$, Anastasija Ziravecka ${ }^{2}$ \\ ${ }^{1}$ Latvian Maritime Academy, Latvia; ${ }^{2}$ Riga Technical University, Latvia \\ aleksandrs.gasparjans@latja.lv, sashater@bkc.lv, zhiravecka@eef.rtu.lv
}

\begin{abstract}
Abstact. The paper contains new results of the investigations announced by the authors in 2017, 2019 in the materials of „The $16^{\text {th }}, 18^{\text {th }}$ International Scientific Conference Engineering for Rural Development”. The work is devoted to determination of the current condition of one of the most loaded elements of the cylinder-piston group of the vessel diesel engines - the piston ring. A new experimental board with a strengthened construction has been built. The piston ring with a gap is placed in a vertical position, it is fixed at a single point symmetrically to the gap. Its both half-segments form a fork. The board has an increased number of magnetoelectric sensors for a simultaneous registration of oscillations of the both segments along the radial and axial surfaces $-\mathrm{X}$ and $\mathrm{Y}$. The oscillations of the ring are investigated in a regime of free and forced oscillations. In the regime of free oscillations, the ring experiences a single impact of a ball with a particular mass, length of its pendant on the angle of the shift. In the regime of forced oscillations, the ring is excited with the undamped oscillations on the frequency of own mechanical resonance and on the even harmonics. The frequency of the oscillations, phase and amplitude depend on the mechanical properties of the ring material and its geometry. The basic frequency of the forced oscillations for the first harmonic is determined experimentally and differs from the theoretical frequency obtained with mathematical modelling for $12-15 \%$. Mechanical oscillations of the ring have larger difference from the theoretical on the higher harmonics with frequency $2 \mathrm{f}_{1}, 3 \mathrm{f}_{1}$. At the first stage of the diagnostics the parameters of the left and right segments are compared with each other. A ring without defects has similar parameters of the segments. The second stage of the diagnostics is the comparison of the obtained results with reference values that are average results of measurements of some serviceable rings without defects.
\end{abstract}

Keywords: diagnostics, resonance, spectrum, harmonics, ring.

\section{Introduction}

This work considers the problems of the theory of oscillations of the open piston rings and the methods of experimental data obtaining. Not very many literature sources are devoted to the research of piston ring oscillations (dynamics) [1-4]. This paper proceeds with those started by the authors before $[5 ; 6]$.

The paper contains the results of the investigation of the dynamic characteristics of a "free" piston ring that is not placed in the grooves of the piston and is not in touch with the walls of the cylinder. The results of the investigations of the dynamics of other elements of the cylinder-piston group will be published later after reliable experimental data are obtained.

If a piston ring is fixed stationary at a point opposite to the gap, then the ring could be approximately considered as a tuning fork. A classical tuning fork of " $U$ " type was invented in 1711 by a court musician of the English Queen John Shore.

The piston rings are one of the most important elements of the central piston mechanism of an engine. They perform three important functions: a) provide a reliable sealing of combustion chamber; b) intensively dissipate the heat from the piston to the cylinder walls; c) regulate the width of the oil film on the inside part of the cylinder mirror. The piston rings operate in hot environment of the aggressive gases with considerably variable loads and differences of temperatures.It is known from the experience of the Riga shipyard that most of the damages of the piston rings are in the region of the gap. With the diameter of the ring 500-800 mm kinks take place mostly at the distance of 50-150 mm from the gap. This is the zone of the increased vibration of the ring. In the piston grooves the ring experiences permanent bending-torsional vibrations.

\section{Materials and methods}

An open piston ring is an oscillating system with distributed parameters. With the first degree of approximation the cross-section area of the piston ring can be assumed very small in comparison with the radius $\mathrm{R}$ of the ring circle. The experimental bench graphically is demonstrated in Fig. 1. 


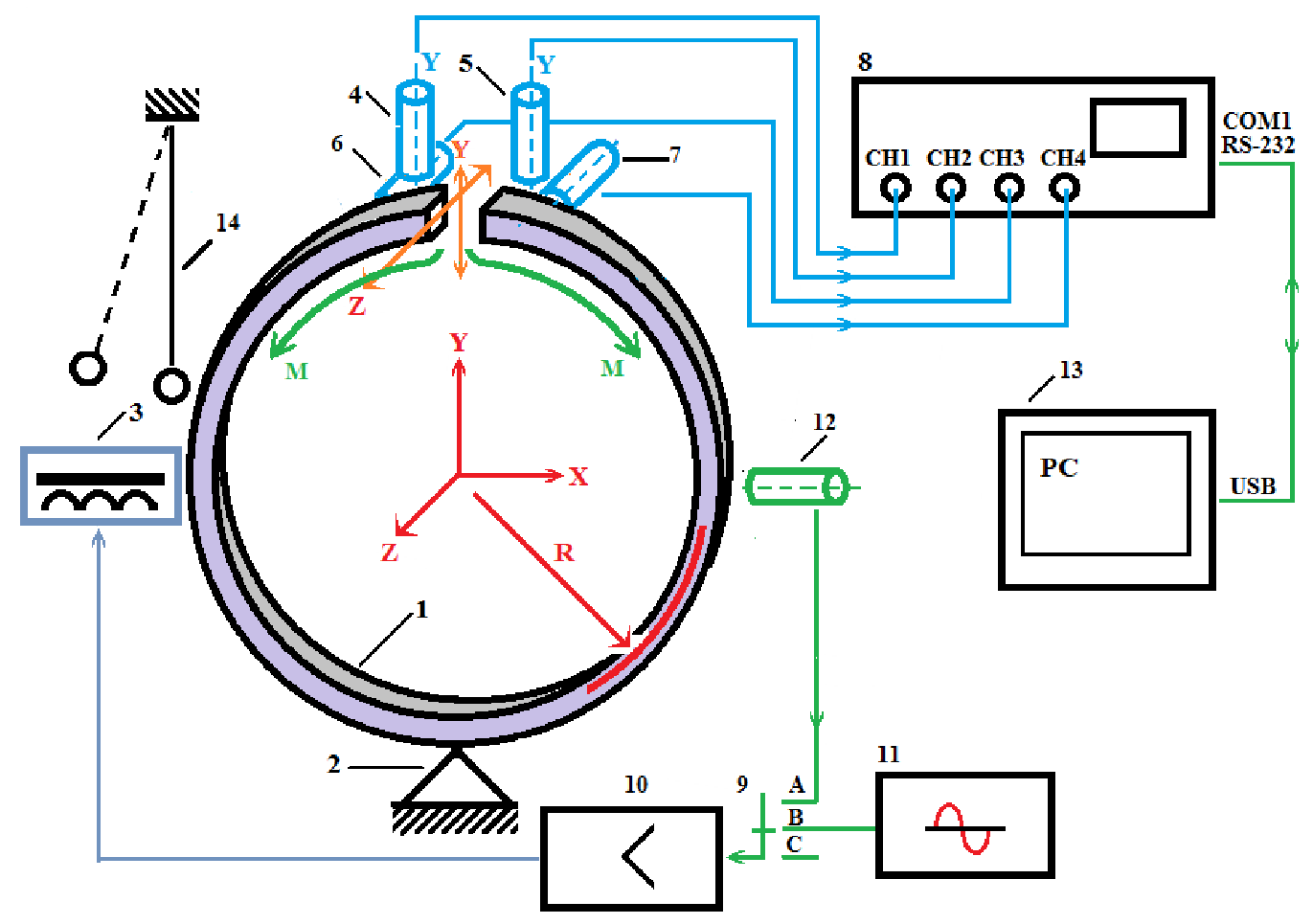

Fig. 1. Experimental bench: 1 - piston ring; 2 - basis; 3 - indignant electro magnet of the feedback loop; 4 and 5 - left $(\mathrm{L})$ and $\operatorname{right}(\mathrm{R})$ magnetoelectric sensors of the ring edges displacements in the radius $\mathrm{R}$ direction, along axis $\mathrm{Y} ; 6$ and 7 left $(\mathrm{L})$ andright $(\mathrm{R})$ magnetoelectric sensors of the ring edges displacements within its own plane, along axis Z; 8 - multichannel oscilloscope - spectrum analyser; 9 - switch of the measurement regimes; 10 - amplifier; 11 - generator of sound; 12 - magnetoelectric sensor of the feedback loop; 13 - computer; 14 - ball. M-bending torque;

$\mathrm{R}$ - radius of the axis line of the ring

A new experimental bench has been developed for the experiments (Fig.2); it has an improved strength of the construction, its mass is increased, own resonant frequency was decreased, the number of the magnetoelectric sensors was increased from 2 to 4 . It allows investigation of the piston rings of different diameters.

Oscillations of the free open ring occur along all three axes $\mathrm{X}, \mathrm{Y}, \mathrm{Z}$. Besides that, the ring experiences bending torques M, Fig. $1[7 ; 8]$.

The piston ring can have grooves of different forms on its outside surface. Fig. 3 demonstrates a fragment of the outside surface of the bottom oil scraping ring. Such form significantly complicates theoretical calculation of the oscillations frequencies. In this experimental bench the frequencies and amplitudes of the oscillations of the both branches of the ring were determined along axes Y andZ. Bending torques $\mathrm{M}$ in this work were not measured. They can be calculated according to the parameters of the oscillations of the ring edges along the axes $\mathrm{Y}$ andZ. If the defects are within the structure of the ring's metal (local zones of the overheating, heterogeneous mechanical stresses, microcracks, etc.), the frequency response characteristics of the both branches of the ring significantly differ one from another - Fig. 5.

This paper specially refers to the self-oscillation regime of the rings (at the frequency of the own mechanical resonance) and forced oscillations of the ring at the frequencies of its harmonics. Free damped oscillations have been considered in the previous articles of the authors [5;6], as well as the correspondent mathematical apparatus for the determination of the parameters of the oscillation process.

The ring can oscillate in its plane (axis $\mathrm{X} 1$ along radius $\mathrm{R}$ ) and perpendicular - along axis $\mathrm{X}_{3}$. With the first degree of approximation the piston ring can be assumed as a ring beam of rectangular section. A real piston ring has a more complicated form. 


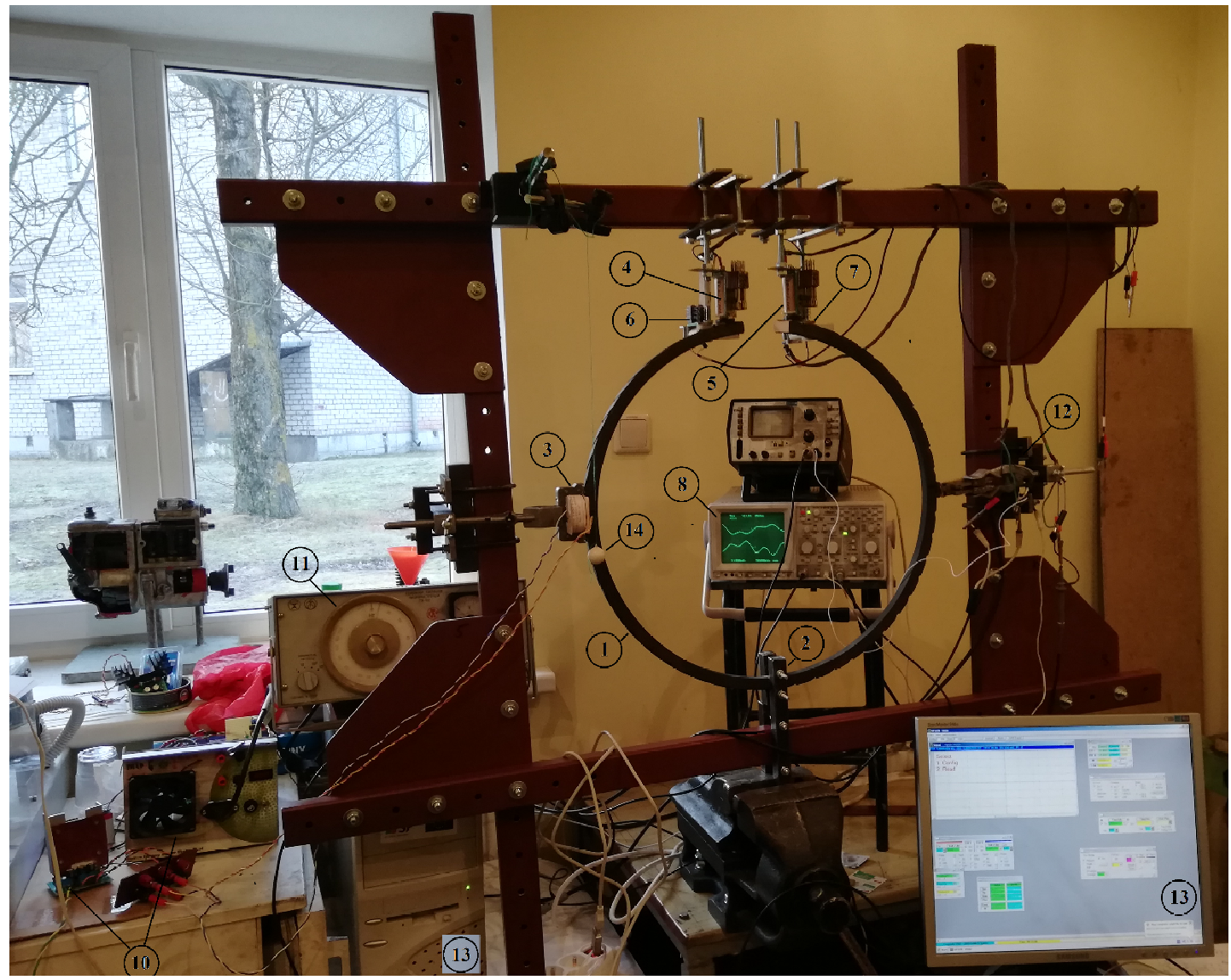

Fig. 2. Picture of the experimental bench (Positions of the elements correspond to those in Fig. 1)

At the calcualtions we assumed that the open ring is inextensible, has a uniformly deistributed mass and constant cross-section.

The applied mathematical methods were considered in more details in [5;6].

\section{Torsional vibrations of the ring.}

From [5] let us get an expression for the frequency $p_{k 1}$ of own torsional oscillations of the ring (along axis $\mathrm{X}_{1}$, Fig.1.):

$$
p_{k 1}=\frac{k\left(k^{2}-1\right)}{\sqrt{k^{2}+1}} \sqrt{\frac{E J}{m R^{4}}},
$$

where $k$ - factor, called mode,

$E J$ - strength of the cross-section at bending;

$E$ - Young's modulus, $\mathrm{Pa}$;

$J$ - moment of inertia of the cross-section according to the central axis, $\mathrm{m}^{4}$;

$m$ - mass, $\mathrm{kg}$;

$R$ - radius of the middle line of the ring, $\mathrm{m}$;

$x$ - displacement of the center of gravity of the ring, $\mathrm{m}$.

The value $k=1$ corresponds to zero frequency.

Oscillations of the ring in the axial direction - along axis Z, Fig. 1.

After the correspondent transformations [5] the own relative oscillations of the ring are calculated $p_{k 2}$ in axial direction (along axis $\mathrm{X}_{3}$, Fig. 1.):

$$
p_{k 2}=\frac{k\left(k^{2}-1\right)}{\sqrt{k^{2} G J_{k} / E J_{1}+1}} \sqrt{\frac{G J_{k}}{m R^{4}}} .
$$


where $J_{1}$ - moment of inertia of the cross-section according to the central axis,within the plane of binding, $\mathrm{m}^{4}$;

$G J_{k}$ - torsional strengtheness of the ring cross-section;

$G$ - modulus of the shift, Pa.

Minimum non-zero frequency is $k=2$.

Investigation of the oscillations of the ring in anself-oscillating regime, at the frequency of own mechanical resonance.

As an example Fig. 3. represents (the upper part of the picture) an oscillogram of the oscillations of the left edge of the piston ring "L-Y"(red line) and right(blue line) "R-Y" edge of the ring in a radial direction along axis Y (Fig. 1)at the frequency of the basic (first) harmonic 19,53 Hzin the regime of self-oscillations.Amplitude-frequency response of these oscillations is shown in Fig. 5 (the bottom part of the picture).

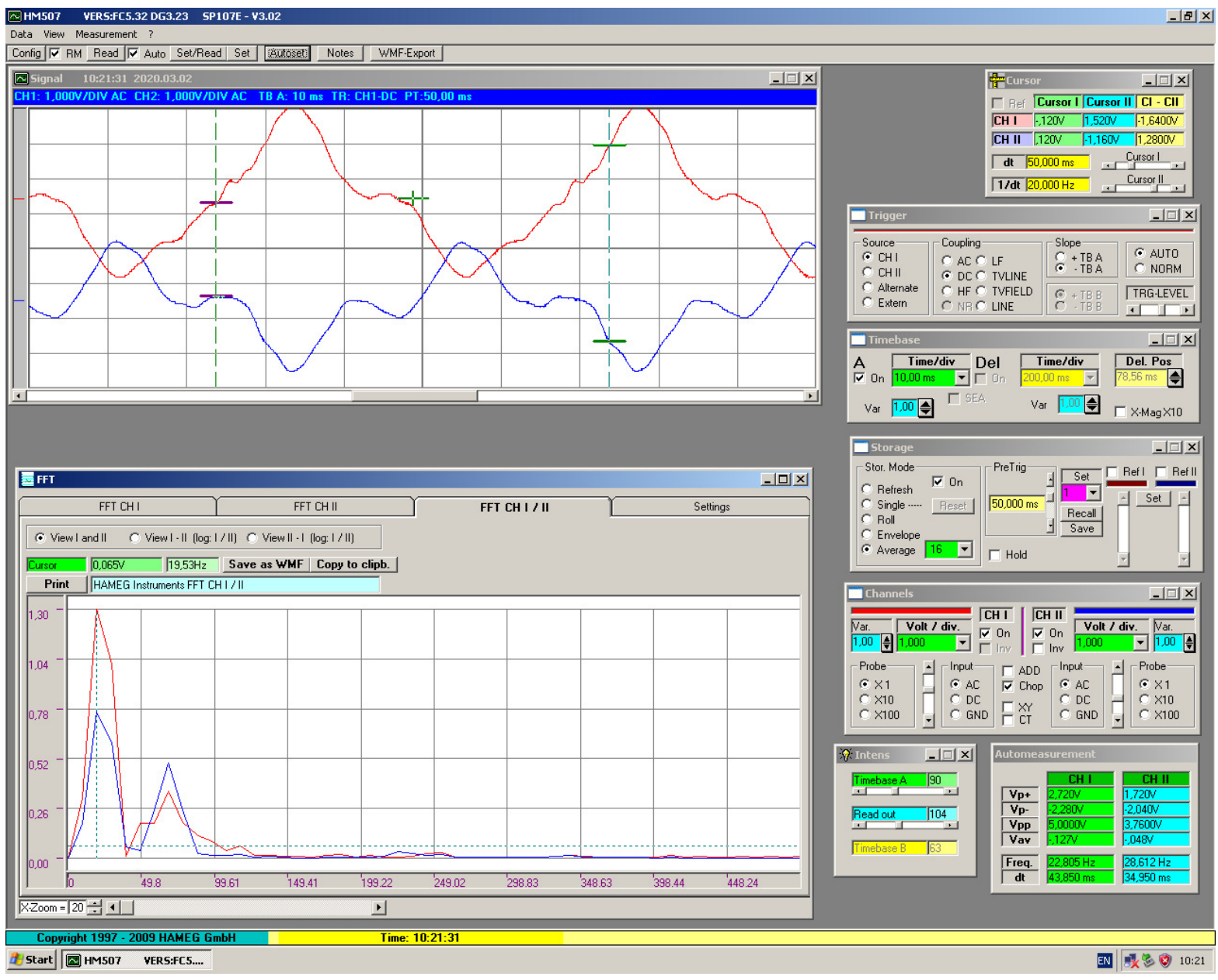

Fig. 3. Form of the oscillations of left "L-Y" and right "R-Y"edges of the ring and spectrum of it (frame No. LY-RY_auto2_19_53_c, 02032020,102300)

From the characteristics it is obvious (Fig. 4) that the spectrum contains higher harmonics - the fourth (frequency $78.12 \mathrm{~Hz}$ ), the twelfth (frequency $234.36 \mathrm{~Hz}$ ), the thirteenth (frequency $253.89 \mathrm{~Hz}$ ).

Fig. 5. demonstrates the amplitude-frequency response of the oscillations of the left "L-Z" and right "R-Z" edges of the piston ring in axial plane along axis $\mathrm{Z}$ in the regime of self-oscillations (basic frequency $19.53 \mathrm{~Hz}$ - the first harmonic $f_{1}$ ).

Fig. 5. shows appearance of harmonics: the third (frequency $58.59 \mathrm{~Hz}$ ), the fourth (frequency $78.12 \mathrm{~Hz}$ ), the eighth (frequency $156.24 \mathrm{~Hz}$ ), the twelfth (frequency $234.36 \mathrm{~Hz}$ ), the twentieth (frequency $390.60 \mathrm{~Hz}$ ).

Fig. 6. represents the amplitude-frequency response of self-oscillations of one of the edges of the piston ring LY in radial (along axis $\mathrm{Y}$ ) andLZ(alogn axis $\mathrm{Z}$ )-in the plane of the ring. 
HAMEG Instruments FFT CH I / II

CII I and CII II
Vindowfuection Hanning - otrdiens. 2020, gads 25, tebruaris 9:23:09

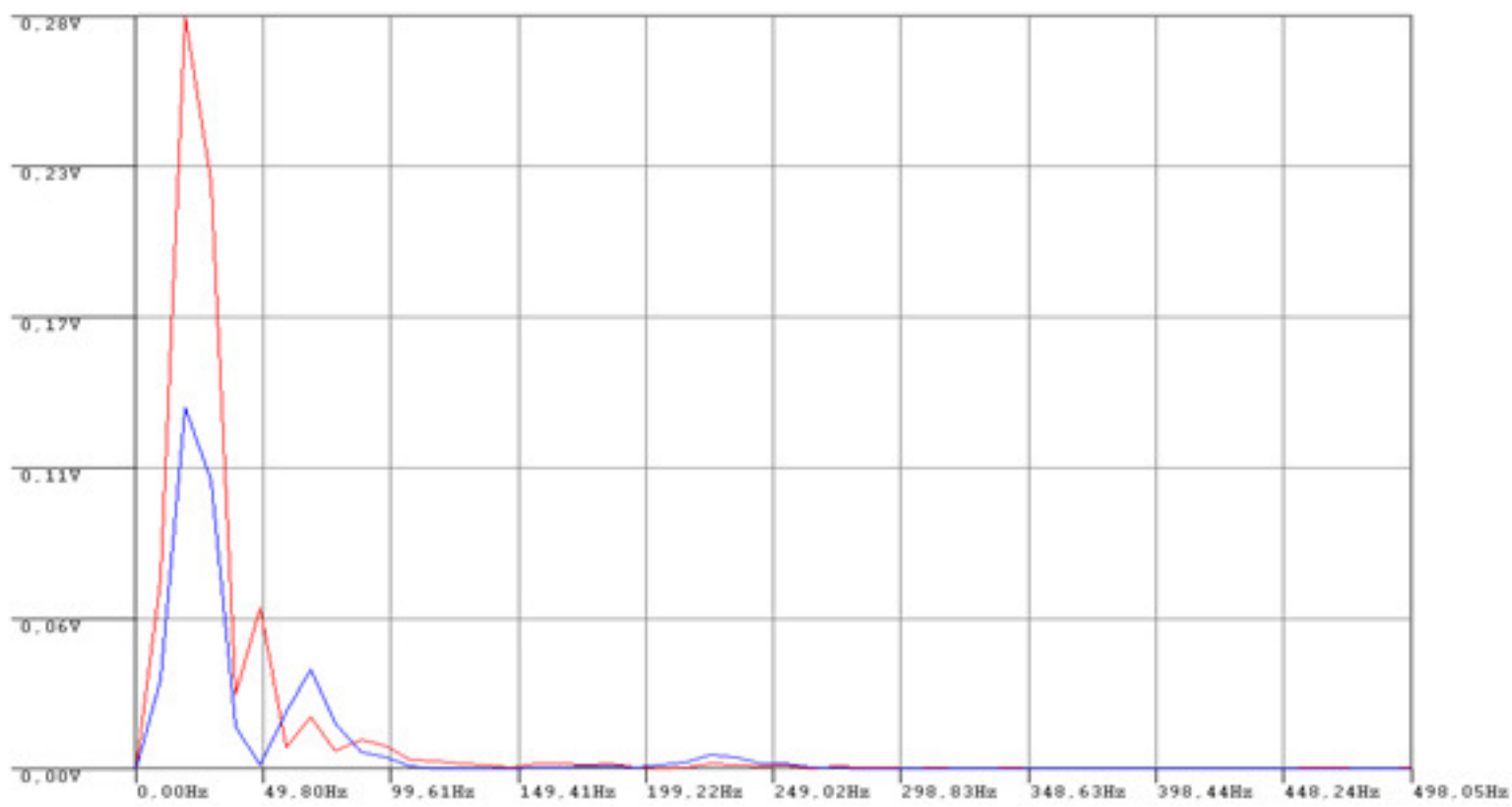

Fig. 4. Amplitude-frequency response of self-oscillations of the left $(L)$ and right $(R)$ edges of the piston ring in the tosional plane (along axis Y) (frame No. LY-RY_auto1_garm3, 25022020, 092309)

HAMEG Instruments FFT CH I / II

CH I and $\mathrm{CH}$ II

Findovfunetion: Bonning -- otrdiens. 2020, gada 25, februaris 10:08:35

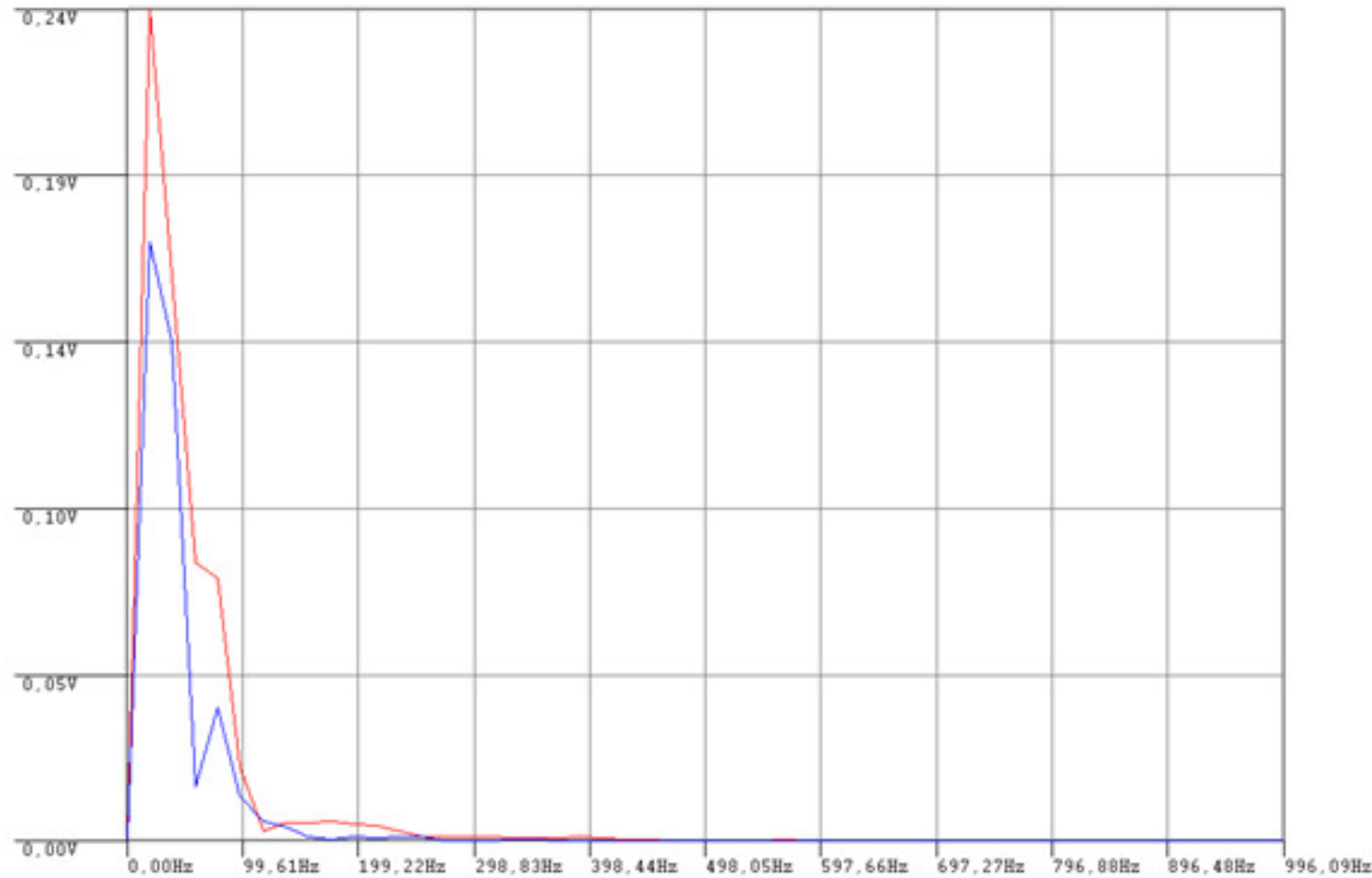

Fig. 5. Amplitude-frequency response of the left and right edges of the piston ring in axial plane (along axisZ)(frame No. LZ-RZ_auto1_garm8, 25022020, 100835) 


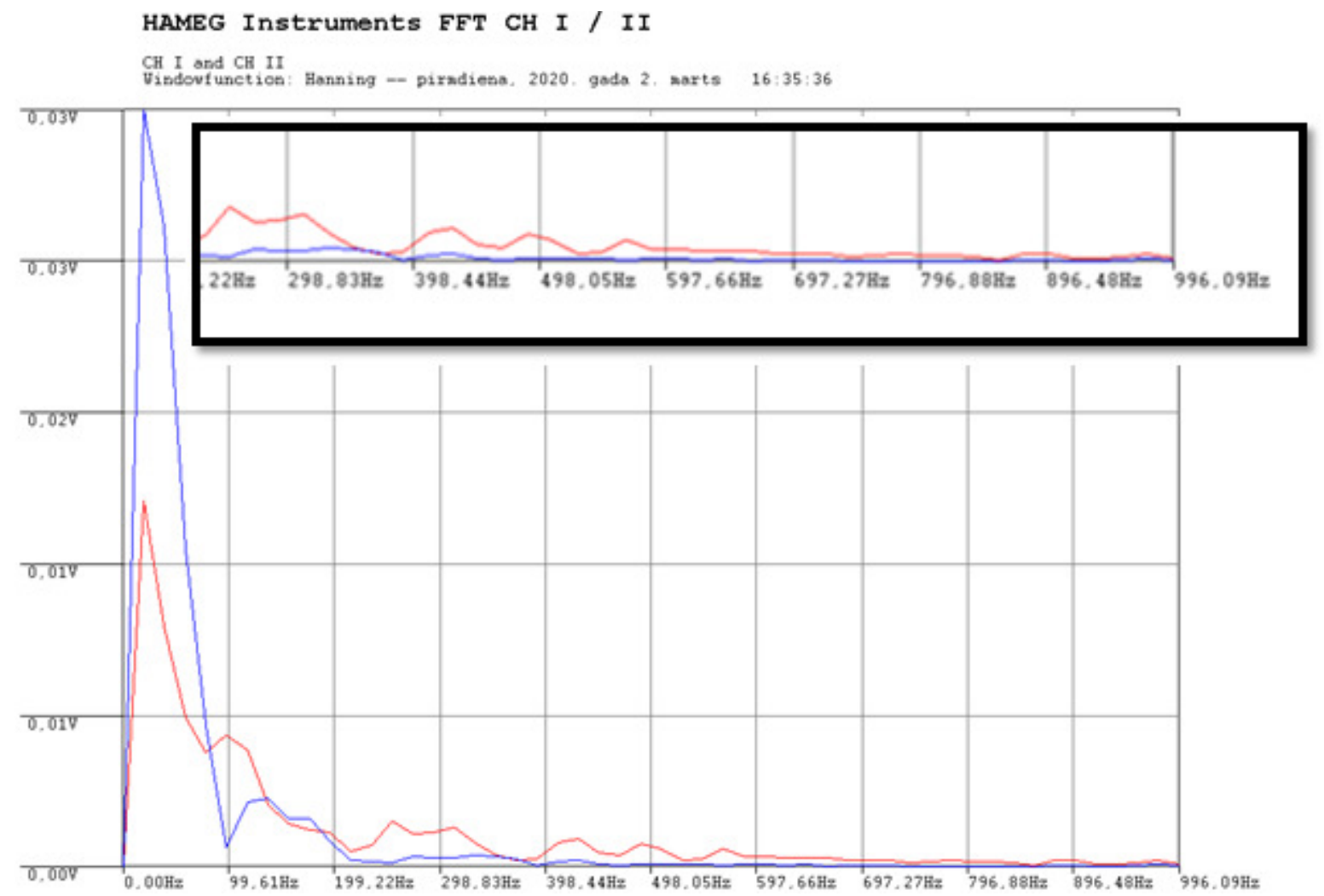

Fig. 6. Amplitude frequency response of one of the edges of the piston ring LY in torsion direction (along axis $Y$ ) andLZ(along axis $Z$ )-in the plane of the ring (FrameLYLZ_auto3_garm_19_53_A, 02032020, 163536), higher harmonics are distributed up to $1000 \mathrm{~Hz}$

The basic frequency of own mechanical oscillations (self-oscillating regime) is $19.53 \mathrm{~Hz}$. From Fig. 3 to Fig. 6 it is clear that the form of oscillations of the right and left edges of the ring are not symmetrical and differ from each other. The amplitude frequency spectrum of the both edges of the ring is also different. It justifies different properties of the left and right branches of the ring. These differences occur due to the presence of unclear defects - thermal heterogeneities, mechanical stresses at some places. This is a diagnostic index of possible defects. In the self-oscillation regime the piston ring runs into steady-state regime of oscillations at own frequency of mechanical resonance $(19.53 \mathrm{~Hz})$ during 2-3 s. When the positive feedback is open (self-oscillations are off), the oscillations are damping approximately during 10-12 s. The time of the damping can be also a diagnostic indicator.

Investigation of the ring oscillation dynamics in the forced regime, at the frequencies of its mechanical harmonics.

In this regime the switch of the regimes 9 was at "B" position (Fig. 1). The positive feedback in this regime is open. The exciting electromagnet 3 through the amplifier 10 gets a signal from the generator of sound 11 . The frequencies of harmonics from the first to the twentieth are set manually on the sound generator (frequencies 19.53-390.60 Hz).

Fig. 7 demonstrates the amplitude frequency of both edges of the ring in torsional direction along axis $\mathrm{Y}$ at the frequency of the sixth harmonic $f_{6}=117.18 \mathrm{~Hz}$. This electromechanical system has a very high quality factor. With a declination of frequency for at least 4-5\%, the amplitude of the oscillations is decreased sharply. At the same frequencies $f_{1}$ and $f_{20}$ in the forced regime the form of the ring oscillations are closer to sine-form than in the regime of self-oscilations. Beside that, the measurements fixed occurrence of sub-harmonics with the period 5 to 7 seconds. Frequency 0.2 to $0.14 \mathrm{~Hz}$.

Fig. 8. contains the amplitude-frequency response of both edges ( $\mathrm{L}$ andR)of the piston ring in torsion direction (along axisY) without defect in the regime of self-oscillations. Harmonics the first $(19 \mathrm{~Hz})$, the second $(39 \mathrm{~Hz})$ and the fourth $(78 \mathrm{~Hz})$ have maximum amplitude. Higher harmonics occur till the frequencies $500 \mathrm{~Hz}$. 


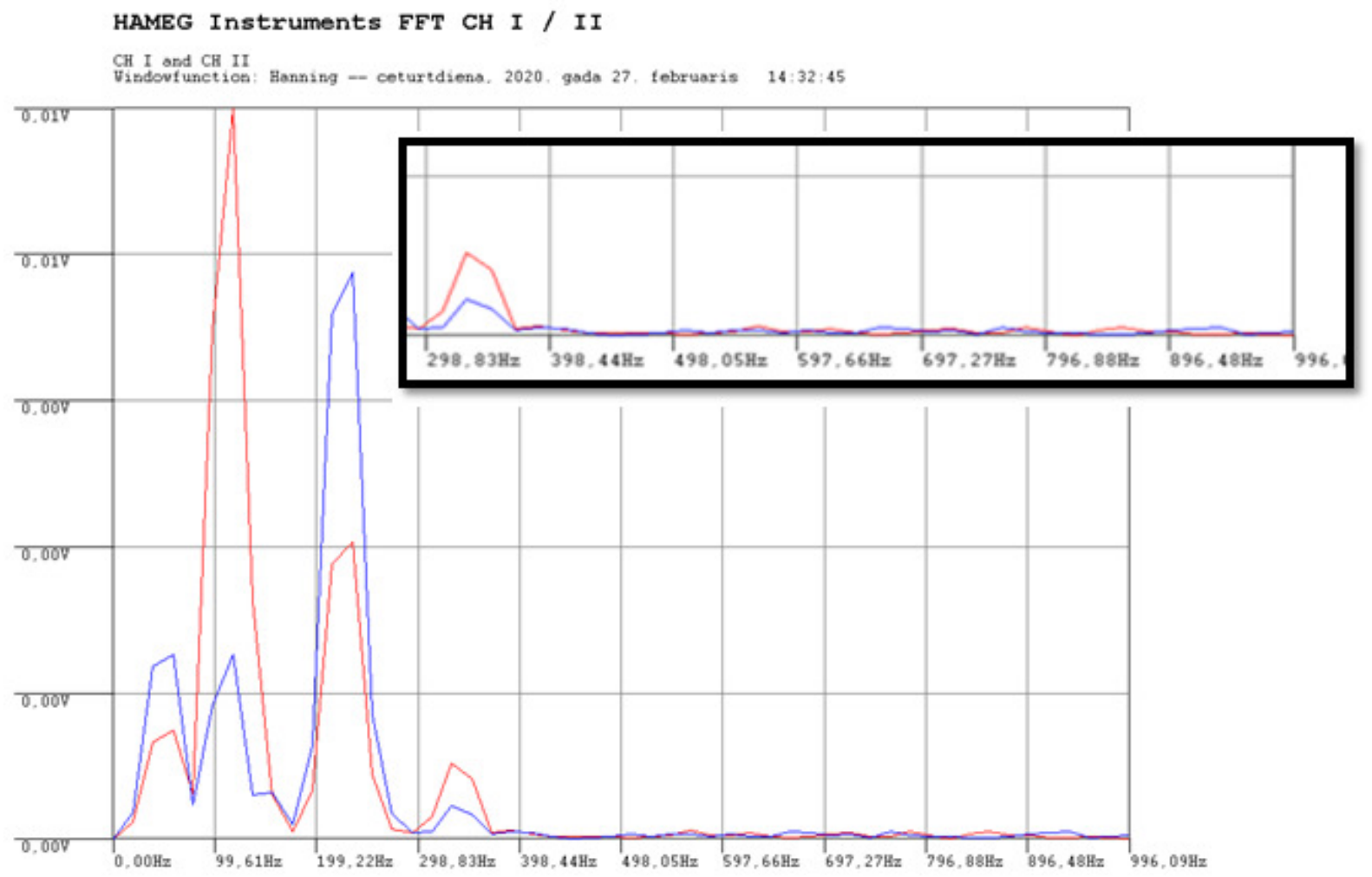

Fig. 7. Amplitude frequency response of the piston ring in torsion direction at the sixth frequency $\mathbf{f}_{6}=\mathbf{1 1 7}, \mathbf{1 8} \mathbf{~ H z}$ (frame No. LY-RY_vinuzhd_117_18. 27022020, 143245)

\section{HAMEG Instruments FFT CH I / II}

CH I and CH II
Findowtunction: Hanning - ceturtdiens, 2020, gada 12, narts 13:50:33

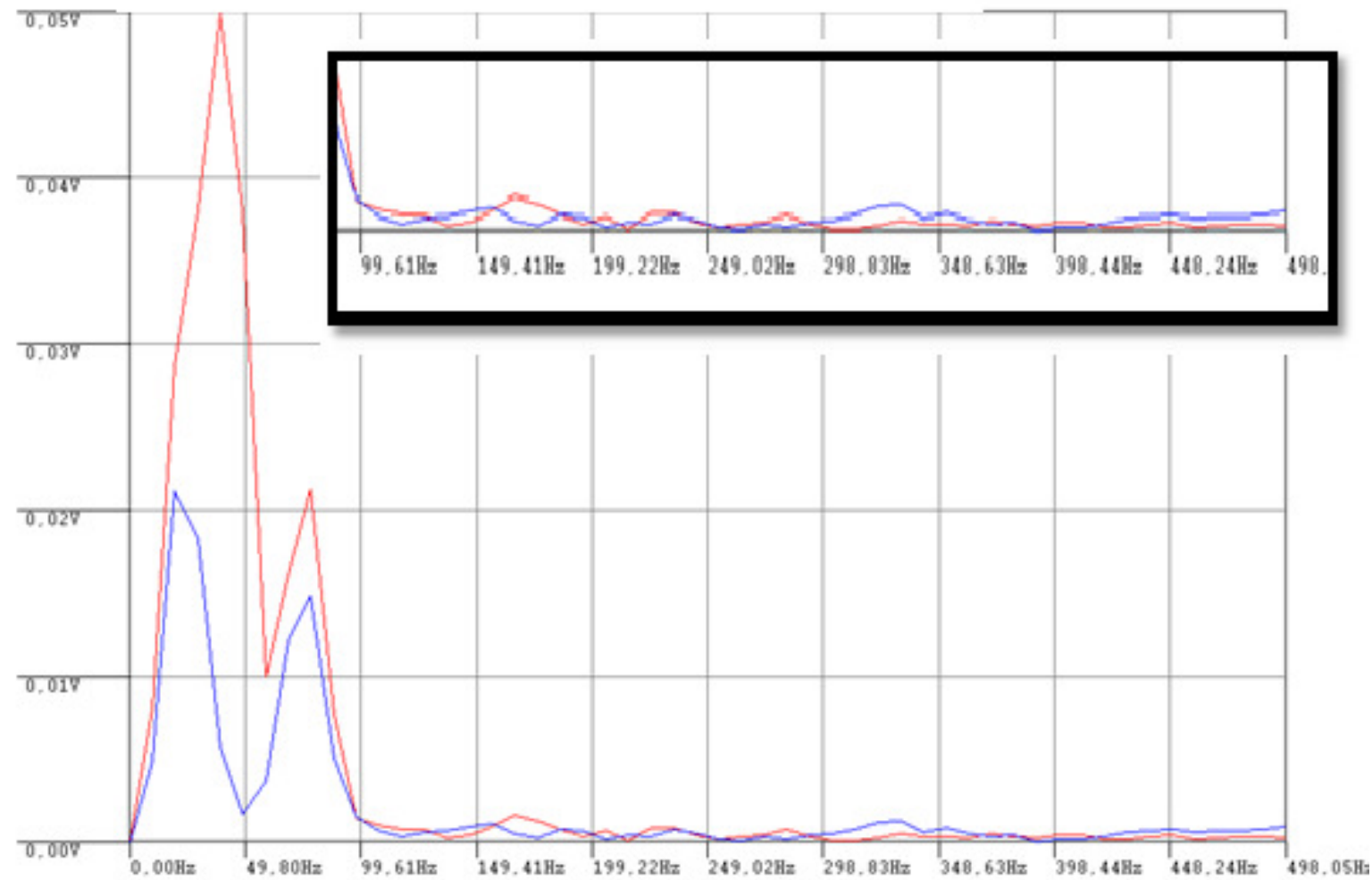

Fig. 8. Amplitude- frequency response of self-oscillations of the piston ring without defects. (frame No. Defek_2_LY-RY_bezgr) 


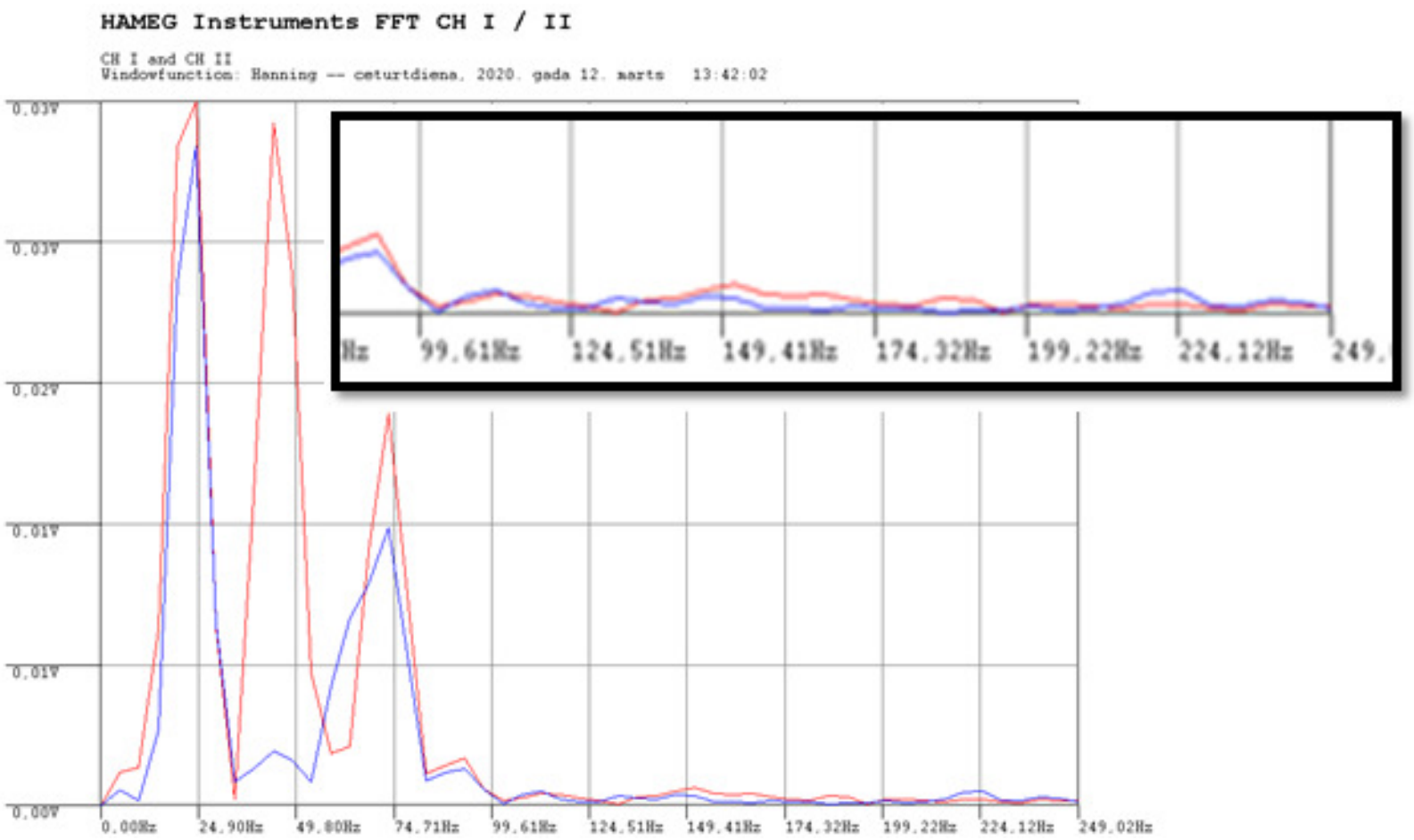

Fig. 9. Amplitude frequency response at a point close to the gap (frame No. Defek_1_LYRY_gruz)

Fig. 9. shows the amplitude frequency response of the same ring with defect. If to compare the characteristics in Fig. 8 and 9, the difference in the spectrums is obvious in frequencies and amplitudes as well. The amplitude frequency response of the ring with defect contains with frequencies $24 \mathrm{~Hz}$, $39 \mathrm{Hzand} 72 \mathrm{~Hz}$. Red line represents the left edge of the ring $\mathrm{L}$, the curve of blue color $-\mathrm{R}$ is the right edge of the ring. Higher harmonics are till $250 \mathrm{~Hz}$. The frequencies of the both branches of the ring are similar. Such properties of the comparison allow an accurate diagnostic of a ring with defect. Having collected large enough amount of statistical material, not only the presence of the defects could be determined, but also their characteristics and location with high enough accuracy.

Table 1

Frequencies of harmonics obtained in experiments and by means of mathematical modeling

\begin{tabular}{|c|c|c|c|}
\hline $\boldsymbol{f}, \mathbf{H z}$ & Harmonic number & $\boldsymbol{U}, \mathbf{m V}$ - exp. & $\boldsymbol{U}, \mathbf{m V}$ - model \\
\hline 19.53 & 1 & 1290 & 1190 \\
\hline 39.06 & 2 & 170 & 175 \\
\hline 58.59 & 3 & 840 & 910 \\
\hline 78.12 & 4 & 1040 & 980 \\
\hline 97.65 & 5 & 10 & 4 \\
\hline 117.20 & 6 & 12 & 4 \\
\hline 136.70 & 7 & 0 & 0 \\
\hline 156.20 & 8 & 25 & 17 \\
\hline 175.80 & 9 & 0 & 0 \\
\hline 195.30 & 10 & 30 & 33 \\
\hline 214.80 & 11 & 15 & 6 \\
\hline 234.40 & 12 & 150 & 165 \\
\hline 253.90 & 13 & 20 & 10 \\
\hline 273.40 & 14 & 15 & 8 \\
\hline 293.00 & 15 & 0 & 0 \\
\hline 312.50 & 16 & 5 & 4 \\
\hline 332.00 & 17 & 0 & 0 \\
\hline 351.50 & 18 & 3 & 4 \\
\hline 371.10 & 19 & 5 & 4 \\
\hline 390.60 & 20 & 13 & 15 \\
\hline
\end{tabular}


Fig. 10 presents the amplitude frequency response of the ring obtained experimentally (blue color) and in modelling (red color). A part of harmonics is fully absent in the experimental characteristics. In this case, the piston ring and exciting electromagnet play a role of narrow bandpass frequency filter with high quality factor.

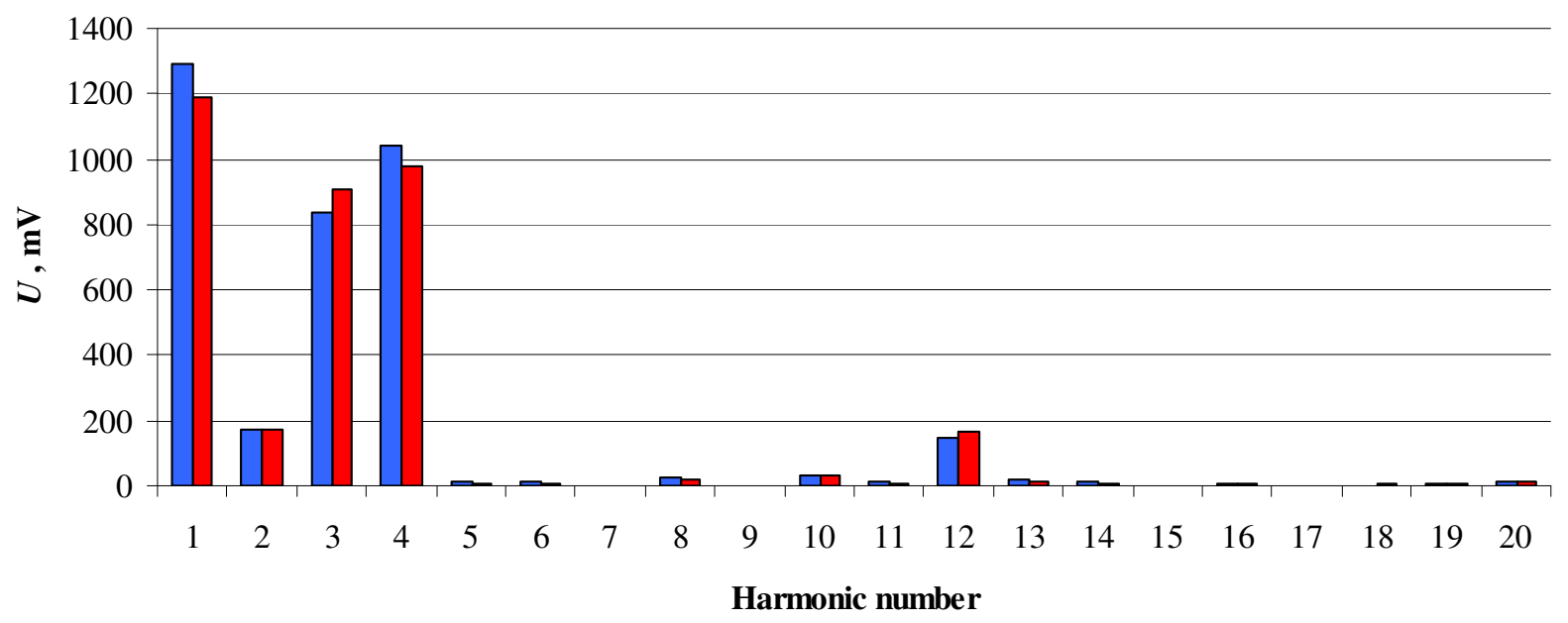

Fig. 10. Amplitude frequency characteristic of the ring oscillations: experimental (blue color) and obtained in modelling (red color)

\section{Results and discussion}

The authors represented the basic results of the investigation with the help of graphs of the amplitude-frequency response and diagrams. There are well-known papers devoted to the research of elastic bodies (tubes of large diameters, elastic tanks, bridge constructions, etc). But, despite the common approaches to the investigation of the oscillation processes, the dynamics of piston rings requires its own specific approach. The proposed method of diagnostics could be applied at the stage of input control before the ring installation onto the piston.About 1-2\% of new rings get defects during the first two-three weeks after the installation under their non-stop operation conditions. The proposed method of the spectral analysis gives an accurate evaluation of the current technical condition of the piston rings. The characteristics can be compared by the mathematical modelling or by the characteristics of some reference rings. The latter is more preferable. The applied measurement equipment should have high sensitivity (from some units of microvolts), high linearity of own characteristics and a low factor of non-liner distortions. Increasing of the amplitude of the existing harmonics, or appearance of new ones, is signaling about the presence or development of unclear defects. Because of complicated form of the oscillations obtained experimentally, the application of digital meters of frequency is difficult - Fig. 8.

\section{Conclusions}

1. The difference of the frequencies obtained experimentally and in modeling is about $15 \%$. In the modelling the assumption was that the ring has a uniform structure and cross-section along all the length of the ring.

2. The proposed method of the spectral analysis gives an accurate evaluation of the current technical condition of the piston rings. Having collected large enough amount of statistical material, not only the presence of the defects could be determined, but also their characteristics and location with high enough accuracy.This approach could be applied together with other non-destructive methods of control.

3. The higher harmonics of even order are the most informative in this method (starting with the sixth and higher).

4. The relatively low value of odd harmonics could be explained by the fact that the branches of the ring have phase oncoming and divergent antiphase oscillations. Increasing of the amplitude and number of the higher harmonics, signals about occurrence of defects in the branches of the ring. 


\section{References}

[1] Naeem M.N., Kanwal S. Vibration Characteristics of Ring-Stiffened Functionally Graded Circular Cylindrical Shells. ISRN Mechanical Engineering. Volume 2012 (2012), 13 p.

[2] Chidamparam P., Leissa A. W.Vibrations of Planar Curved Beams, Rings, and Arches. ASME theAmerican Society of Mechanical Engineering. Appl. Mech. Rev 46(9), 2009, pp. 467-483.

[3] Kosicin A.V. Method of vibrodiagnostics of the elastic constructions on the basis of own oscillations analysis, № 2 (3), 2011. Navy Academy of the Republic of Belarus. pp. 129-135. [online][11.02.2020] Available at: https://cyberleninka.ru/article/v/metod-vibrodiagnostikidefektov-uprugih-konstruktsiy-na-osnove-analiza-sobstvennyh-form-kolebaniy (In Russian).

[4] Naeem M. N., Arshad S. H., Sharma C. B. The Ritz formulation applied to the study of the vibrationfrequency characteristics of functionally graded circular cylindrical shells. Proceedings of the Institution of Mechanical Engineers Part C, vol. 224, no. 1, 2010, pp. 43-54.

[5] Gasparjans A., Terebkovs A., Ziravecka A. Application of resonance method for examining of piston ring Technical condition.16th International Scientific Conference, University of Agriculture Faculty of Engineering.Engineering for rural Development. Proceedings, Volume 16, ISSN 1691-5976, May 24-26, 2017, Jelgava, Latvia. pp. 884-891.

[6] Gasparjans A., Terebkovs A., Ziravecka A. Application of resonance method for examining of piston ring technical condition.16th International Scientific Conference,University of Agriculture Faculty of Engineering.Engineering for Rural Development. Proceedings, Volume 16, ISSN 1691-5976, May 24-26, 2017, Jelgava, Latvia. 884-891 pp.

[7] Колебания упругих тел (Oscillations of elastic bodies) [online][11.02.2020] Available at: https://mash-xxl.info/page/065180192114246028030142113053218196102090032098/ (In Russian).

[8] Изгиб колец (Bending rings) [online][11.02.2020] Available at: http://scask.ru/c_book_brg.php?id = 197 (In Russian). 